\title{
AC 2007-1062: ONLINE COMPUTER SIMULATION TOOLS FOR DIPOLE ANTENNA RADIATION PATTERNS
}

\section{Adam Neale, University of Waterloo}

Adam $\mathrm{J}$ Neale is currently working towards the B.A.Sc. degree in Honours Electrical Engineering at the University of Waterloo, Waterloo, ON, Canada. His interests lie in the area of hardware development using FPGA's as well as student government. He is currently Vice President Internal for the undergraduate Engineering Society at the University of Waterloo.

\section{Jason Shirtliff, University of Waterloo}

Jason N Shirtliff is currently working towards the B.A.Sc. in Honours Computer Engineering at the University of Waterloo, Waterloo, ON, Canada. His interests include VLSI, mixed signal integrated circuit design, and digital application specific integrated circuit design. He was employed for eight months at the Microsoft Online Learning Initiative where he worked on lab development for courses related to microprocessor systems and interfacing and antenna design.

\section{William Bishop, University of Waterloo}

Dr. William Bishop obtained his PhD in Electrical and Computer Engineering from the University of Waterloo in Waterloo, Ontario, Canada. Bill is currently a full-time lecturer in the Department of Electrical and Computer Engineering at the University of Waterloo. His research interests include configurable computing, tools and strategies for e-learning, and image and video processing.

\section{Cutberto Santillan Rios, University of Waterloo}

Cutberto A Santillan received the Engineering degree in Electronic and Communications from the Instituto Tecnologico y de Estudios Superiores de Monterrey, Mexico City, in 1999 and the M.A.Sc. in Electrical and Computer Engineering from the University of Waterloo, in 2002. He is also working towards his $\mathrm{PhD}$ degree in the same institution. He is currently working as a Laboratory Instructor for electromagnetic, communications and electronic circuit design courses at the University of Waterloo. His research interests include RF \& Microwave design, measurement and analysis, RFICs, electronic circuit design and antenna modeling. 


\title{
Online Computer Simulation Tools for Dipole Antenna Radiation Patterns
}

\begin{abstract}
Interactive computer simulation tools are an essential component of a modern pedagogy for electrical and computer engineering. Simulation tools offer dynamic, interactive, self-paced learning that is available at the convenience of the student. At the University of Waterloo, Waterloo, ON, Canada, all senior level undergraduate students studying electrical engineering are required to take a course on electromagnetic waves to satisfy their degree requirements. The final laboratory exercise associated with this course requires students to measure, record, and analyze a series of dipole antenna radiation patterns for various antenna configurations. A common conceptual challenge for students to overcome when dealing with radiation patterns is the full effect of how the three dimensional field changes based on the antenna's configuration parameters.
\end{abstract}

To counteract this issue, our university developed a collection of four dipole antenna radiation pattern simulation tools specifically tailored for the course. Comparable simulation tools that fit the needs of the course cannot be found online. The simulation tools display patterns for: a single dipole, an array of dipoles, a dipole above an infinite flat ground plane, and a dipole inside of a $90^{\circ}$ infinite corner reflector. Students use the simulators to complete a pre-laboratory study to improve their understanding of the material, and to better utilize laboratory experimentation time. The online simulations supplement traditional lectures and laboratory experience by providing a deeper understanding of the concepts using online learning resources.

The simulation tools were first incorporated into the course during the spring 2006 term, and will next be used during the spring 2007 term. Although a comprehensive study into the effectiveness of the simulators has not been completed, the initial feedback from students has been favourable. These tools are now available online at the university's website to members of the Microsoft Developer Network Academic Alliance (MSDN AA). The simulators were developed using Microsoft Visual Studio .NET 2003 using the C\# programming language.

\section{Introduction}

The visualization of electromagnetic radiation can be remarkably difficult for undergraduate students in electrical engineering programs. This is particularly true for students studying electromagnetic phenomena for the first time. Introductory courses on antenna theory pose a significant challenge for undergraduate students. In some situations, students may not have access to a safe lab environment and the equipment necessary to experiment with antenna radiation patterns. Thus, good visualization tools are desirable for use in an introductory course on antenna theory.

Current textbooks are limited in that they only possess the ability to display static images of antenna patterns at a limited number of fixed parameters. To examine the effects of parameter changes, students must perform a series of long and tedious calculations just to plot a single 
point in a radiation pattern. The calculations necessary to plot an entire radiation pattern can be quite time consuming. Wentworth attempts to overcome this difficulty through the supplementation of MATLAB simulations, however these simulations are limited in that they provide a non-interactive movie rather than a fully dynamic tool ${ }^{1}$. When students find it difficult to grasp how changes in antenna parameters affect the resulting radiation patterns, students find it difficult to develop an intuitive understanding of the material. Without this understanding, they have no way to check their work. A scenario such as this lends itself easily to a computer simulation solution where students have the ability to alter simple antenna parameters to instantly see the resulting effect without the need to perform all of the required calculations.

Fourth year electrical engineering students at the University of Waterloo, Waterloo, ON, Canada must take a compulsory course on electromagnetic waves (ECE 471) to complete their program. This course covers topics on electromagnetic theory such as wave theory, waveguide structures, microwave circuit analysis, radiation patterns, and antenna theory. The course is complemented by a few practical laboratory sessions. Additionally, ECE 471 serves as a pre-requisite for a fourth year technical elective course on antennas and wireless systems (ECE 476). To prepare students for the elective course, the fourth laboratory study of ECE 471 examines antenna theory. The goal of this lab study is to measure, simulate, and calculate the antenna radiation patterns for a single half-wave dipole antenna with and without the presence of a metallic corner reflector. To prepare students for this laboratory exercise, the teaching team decided that an online, interactive simulation tool was needed. Such a tool could be used as a pre-lab exercise to help students gain a deeper understanding of dipole antenna radiation patterns.

After an in-depth online investigation, a limited number of potential simulation tools were found $^{2-5}$. However, none of the available tools accurately reflected the laboratory material enough to warrant use in the course. Most of the existing simulators either did not use the desired antenna configurations or did not facilitate altering the desired parameters of interest. The tools that did allow the detailed examination of the desire parameters of interest were too complicated and contained too many features for the simple pre-lab exercise. Also, none of the tools attempted to explain the underlying theory in a way suitable for students. Thus, it was determined that a simulation tool should be developed by the university to facilitate the needs of the dipole antenna laboratory study.

The end product of this development is a suite of four simulation tools integrated into a single program through a simple menu system. The four simulators display patterns for a single dipole antenna, an array of dipole antennas, a single dipole antenna horizontally oriented above an infinite flat ground plane, and a single dipole antenna situated inside of a $90^{\circ}$ infinite corner reflector. Each one of these simulators displays both the azimuth and elevation radiation patterns for a given set of parameters using a pair of two-dimensional polar coordinate grids. Students are permitted to change each antenna configuration's parameters and observe the resulting radiation pattern change in real-time. Each simulator comes complete with a set of controls specific to the antenna configuration under study, a physical representation of the antenna that changes dynamically along with the pattern, and a print function capable of printing the set patterns along with all of the relevant parameter values. The tools were developed using Microsoft Visual Studio .NET 2003 and the C\# programming language. 


\section{Design and Development}

Figure 1 illustrates the single dipole antenna tool. It is the simplest of the simulation tools. Its unique features are explained in the features section below, but is provided here as a visual aid for explaining the features common to each simulator.

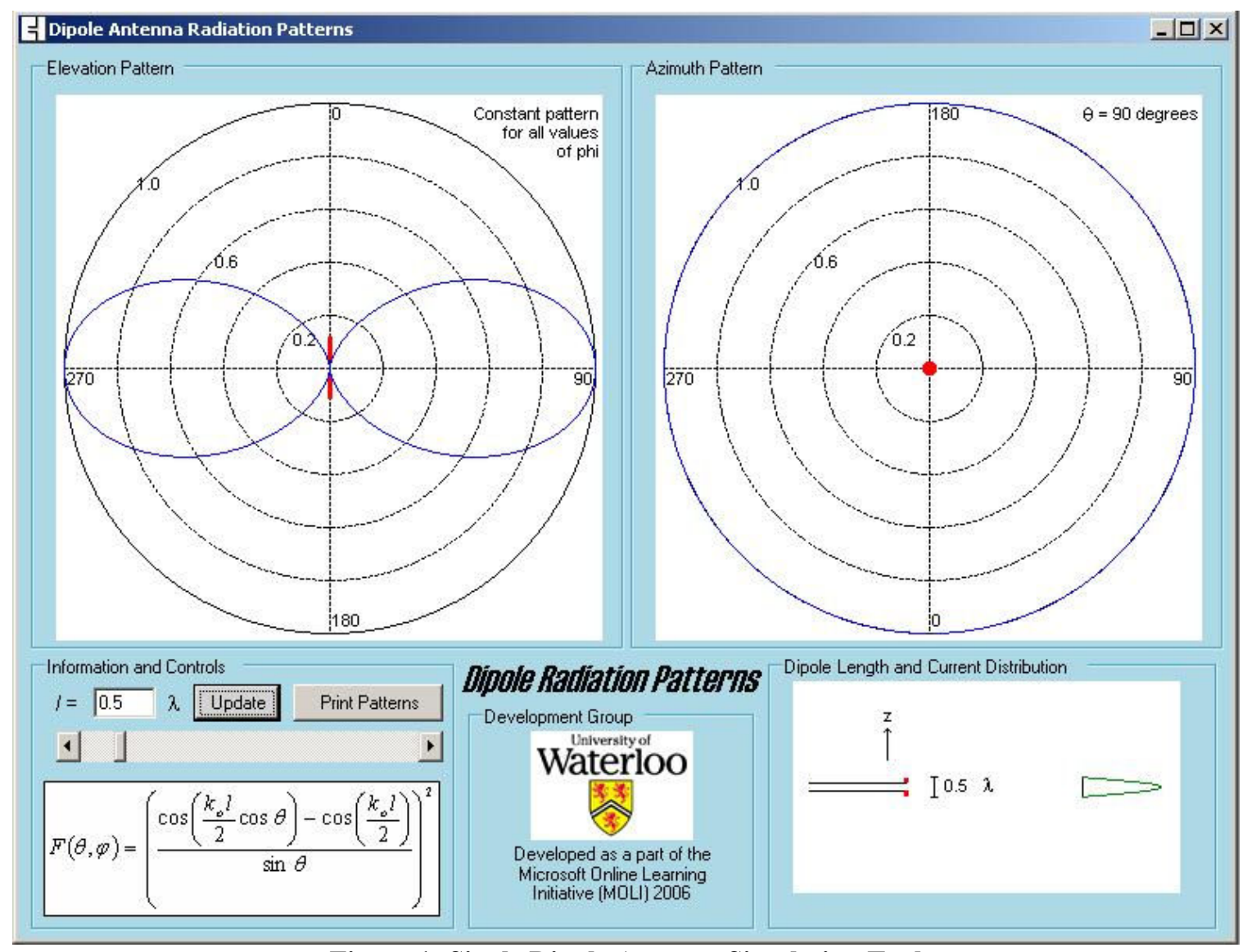

Figure 1: Single Dipole Antenna Simulation Tool

The common layout for each of the antenna simulation tools consists of three main sections. The field viewing area consists of a pair of two-dimensional polar coordinate grids capable of displaying the azimuth and elevation patterns for the antenna given a set configuration. The elevation pattern, on the left hand side of each simulator, displays the electric field. The azimuth pattern, on the right hand side of each simulator, displays the magnetic field. By mentally putting these two images together, a single three-dimensional pattern can be realized by the student. In Figure 1, the elevation pattern can be interpreted as a figure-eight shape. This pattern can be thought of as a side-view of the overall pattern. The azimuth pattern in Figure 1 is circular in shape. This is an overhead or bird's eye view of the pattern. These two shapes, when put together, can be interpreted as something of a donut-shape for the single dipole antenna at a length of $0.5 \lambda$, where $\lambda$ is the wavelength of the electromagnetic wave measured in meters. All of the radiation patterns have been normalized to the antenna's maximum range. 
The second section is the user controls section that contains all of the user operable controls for changing the parameters of the dipole antenna configuration. These controls are different for each simulation tool since each scenario requires a custom set of controls.

The final section is the physical representation window. It displays the physical geometry of the antenna's configuration as it would appear in real life. By providing this physical representation, a student gains an understanding of the physical appearance of the antenna based on the parameters entered. By providing this visualization, students learn what the parameters actually mean in the "real world". Within the physical representation window, the current distribution along the dipole antenna is also shown alongside the dipole graphic. This provides students with a means to investigate current distribution changes along the dipole as a function of the antenna's length.

All of the simulation tools use a fixed window size of $800 \times 600$ pixels. This allows for the simulators to be properly viewed on student's computers that use lower resolution settings. Finally, the executable files for the simulation tools are contained within a single compressed zip file that may be downloaded to a personal computer. The downloadable file, containing all four simulators, the main menu, and complete documentation has a file size of $337 \mathrm{kB}$, making downloading the file manageable even for students without a high-speed Internet connection.

\section{Variable Parameters}

The variable parameters within the user controls section for each simulator are summarized in Table 1. Each of the parameters is described in further detail in the features section for each antenna configuration below.

Table 1: Variable Parameters

\begin{tabular}{|l|l|}
\hline \multicolumn{1}{|c|}{ Configuration } & \multicolumn{1}{c|}{ Parameter(s) } \\
\hline Single Dipole & Length of dipole (in terms of $\lambda$ ) \\
\hline \multirow{4}{*}{ Dipole Array } & Length of dipole (in terms of $\lambda$ ) \\
\cline { 2 - 2 } & Distance between dipoles (in terms of $\lambda$ ) \\
\cline { 2 - 2 } & Number of dipoles (3, 5, or 7) \\
\cline { 2 - 2 } $\begin{array}{l}\text { Single Dipole above a Flat } \\
\text { Ground Plane }\end{array}$ & Weighting of dipoles (Linear, Binomial, or Exponential) \\
\cline { 2 - 2 } & Pattern relative to single dipole antenna \\
\hline \multirow{2}{*}{$\begin{array}{l}\text { Single Dipole Inside a } 90^{\circ} \\
\text { Corner Reflector }\end{array}$} & Length of dipole (in terms of $\lambda$ ) \\
\cline { 2 - 2 } & Pattern relative to single dipole antenna \\
\cline { 2 - 2 } & Length of dipole (in terms of $\lambda$ ) \\
\cline { 2 - 2 } & Distance from ground (in terms of $\lambda$ ) \\
\cline { 2 - 2 } & Pattern relative to single dipole antenna \\
\hline
\end{tabular}

\section{Single Dipole Antenna Features}

The single dipole antenna simulation tool, shown in Figure 1, is the simplest of all the antenna simulation tools. The only parameter available for students to change is the length of the dipole in terms of wavelength. Students are permitted to enter a specific value for the length in the 
input box provided. Alternatively, students may linearly increase or decrease the value by use of a slider. When the student changes the length parameter, the tool automatically updates the pattern viewing areas and the physical configuration window.

\section{Dipole Antenna Array Features}

The second simulation tool allows users to view the effect of having multiple equidistant dipoles in a linear array configuration. This tool is illustrated in Figure 2.

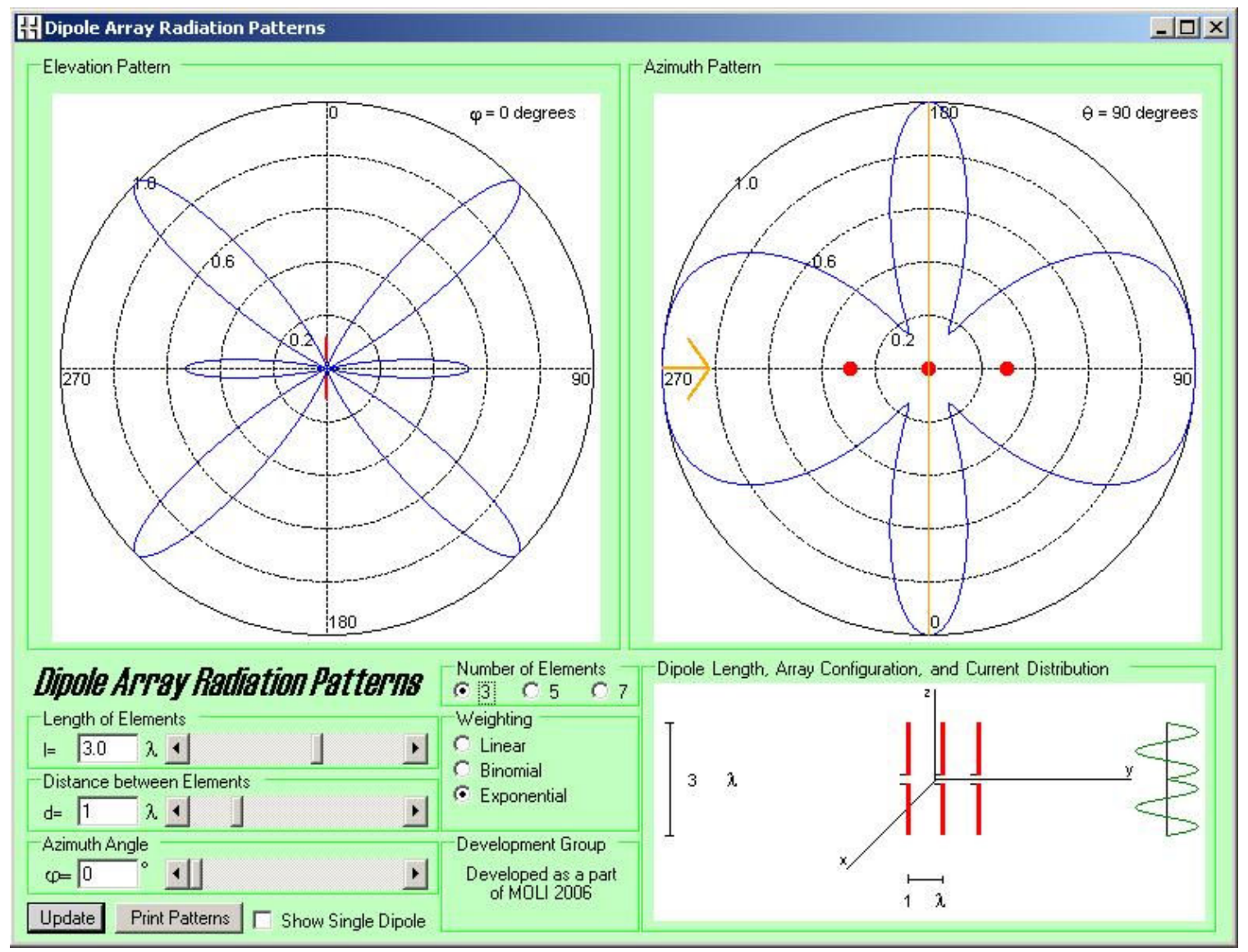

Figure 2: Dipole Array Simulation Tool

This tool is more feature rich than the single dipole antenna tool. The tool provides six different parameters for the user to alter. Similar to the single dipole simulator, users can change the uniform length of the dipole elements. Users may also vary the number of dipole antenna elements in the array. This feature is limited to a choice of 3, 5, or 7 elements since these are the configurations used in the lab studies in the course. As well, the distance between the dipoles can be set; again this is uniform since the dipoles are equidistant. The weighting of the elements can be set to linear, binomial, or exponential weighting. This weighting refers to each element's contribution to the overall signal strength of the electromagnetic wave. The weightings are provided in Table 2 . 
Table 2: Element Weighting

\begin{tabular}{|c|c|c|c|c|}
\hline \multirow{2}{*}{ Weighting } & \multicolumn{4}{|c|}{ Distance from Center Element (Number of Elements) } \\
\cline { 2 - 5 } & Center (0) & $\mathbf{1}$ & $\mathbf{2}$ & $\mathbf{3}$ \\
\hline Linear & 1 & 1 & 1 & 1 \\
\hline Binomial & 1 & 0.5 & 0.25 & 0.125 \\
\hline Exponential & 1 & $e^{-1}$ & $e^{-2}$ & $e^{-3}$ \\
\hline
\end{tabular}

For the linear weighting, each element in the array contributes to the signal strength equally, and therefore has a weighting of unity. For the binomial weighting, the weighting of each element is calculated using Equation 1.

$$
\operatorname{Weight}(m)=\frac{1}{2 * m} \quad \text { If } \mathrm{m}>0, \text { and } 1 \text { if } \mathrm{m}=0
$$

The variable $m$ represents the $m^{\text {th }}$ dipole element from the center reference element of the array. For instance in the case of a five element array, the $0^{\text {th }}$ center element would have a weighting of 1 , the two elements immediately adjacent the center element would have a weighting of $1 / 2$, and finally the elements outside of those would have a weighting of $1 / 4$. For the exponential weighting, the weighting of each element is calculated using Equation 2.

$$
\operatorname{Weight}(m)=e^{-m}
$$

As with the binomial weighting, the exponential weighting $m$ represents the $m^{\text {th }}$ element away from the origin of the configuration. The element at the origin has a weighting of unity, and the weighting of the subsequent elements decay exponentially away from the center of the array.

The azimuth angle for the elevation pattern can be set as well. Since the two-dimensional pattern only displays a single slice of the radiation pattern along the azimuth angle, by changing that angle, the user has the ability to rotate around the elevation radiation pattern to view the pattern as it varies with azimuth angle. The elevation pattern viewing angle can be taken into perspective by noting the position of the indicator line and arrow on the azimuth pattern display. The arrow indicates the observer's point of reference, and the line indicates the azimuth angle slice along which the elevation pattern is being viewed. Finally, the dipole array simulator is also able to compare the radiation pattern of the array configuration relative to a single dipole configuration with the same dipole length. When this feature is enabled, the single dipole pattern is overlaid on the viewing grids with the array configuration pattern, and both patterns are normalized to the wider ranging of the two patterns. This ensures that both of the patterns stay on the grid, and a relative comparison can be obtained. As the parameters for the number, length, and spacing of dipoles are altered, this information, as well as the current distribution along the dipole, is updated in the physical configuration window.

\section{Dipole Antenna above a Infinite Flat Ground Plane Features}

The third simulation tool simulates the radiation pattern displayed in the case of a single dipole antenna positioned horizontally above an infinite flat ground plane. This tool is illustrated in Figure 3. 


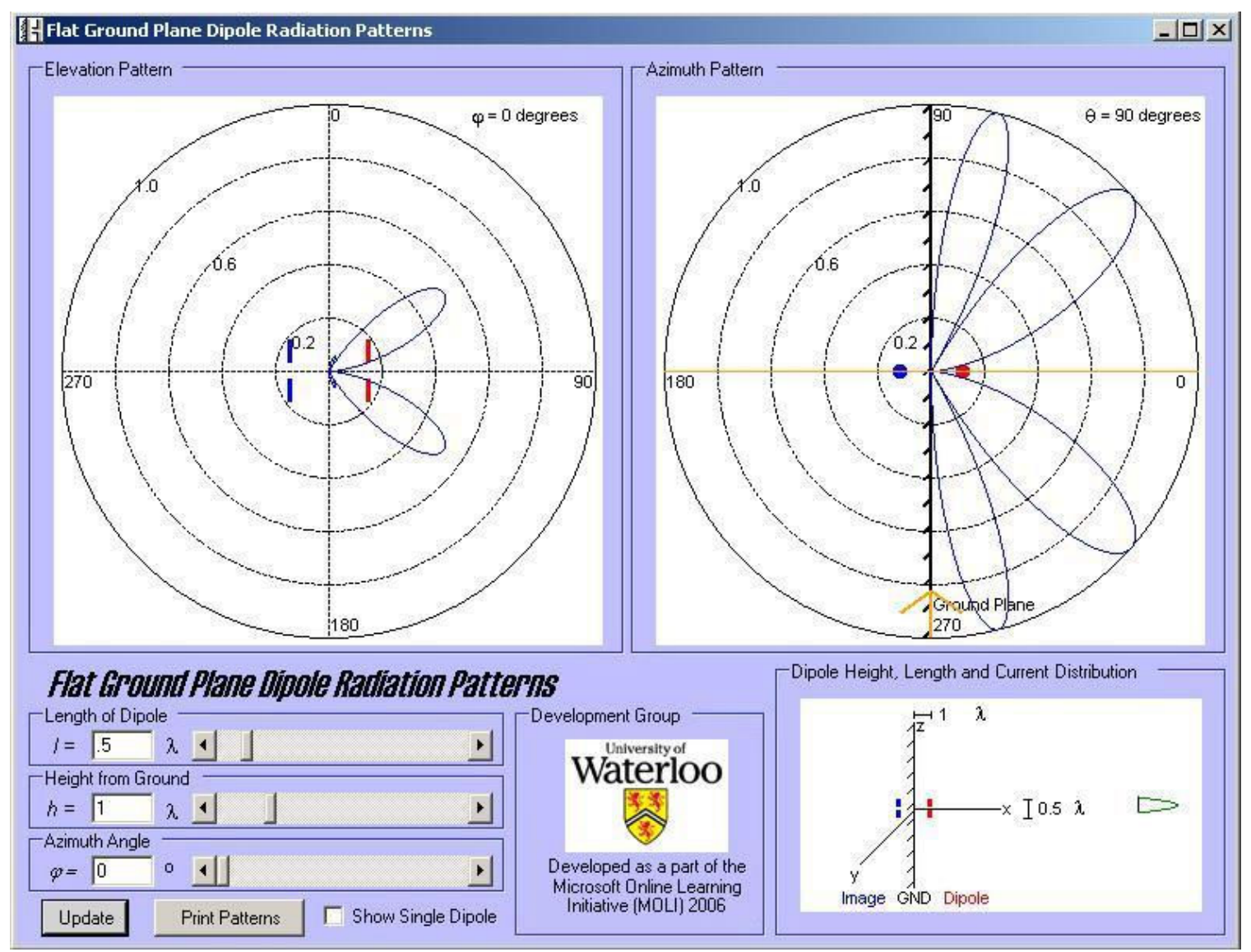

Figure 3: Single Dipole above an Infinite Flat Ground Plane Simulation Tool

In this case, an image of the dipole is produced on the opposite side of the ground plane. This image is signified in blue on the display grid and the physical representation window. This image acts as a second dipole, but its effects are only seen on the side of the ground plane containing the actual dipole. For this simulator, the user has the ability to alter the length of the dipole, the azimuth angle, and the dipole's height above the ground plane, and compare the radiation pattern to that of a single dipole. By altering the height from ground, the dipole image is also moved the same distance from the ground plane on the opposite side of the plane. The length of the dipole, its height from the ground plane, and the current distribution along the dipole are displayed in the physical configuration window.

\section{Dipole Antenna in a $90^{\circ}$ Corner Reflector Features}

The fourth and final simulation tool simulates the radiation pattern of a single dipole antenna situated inside of an infinite $90^{\circ}$ corner reflector. This tool is illustrated in Figure 4. 


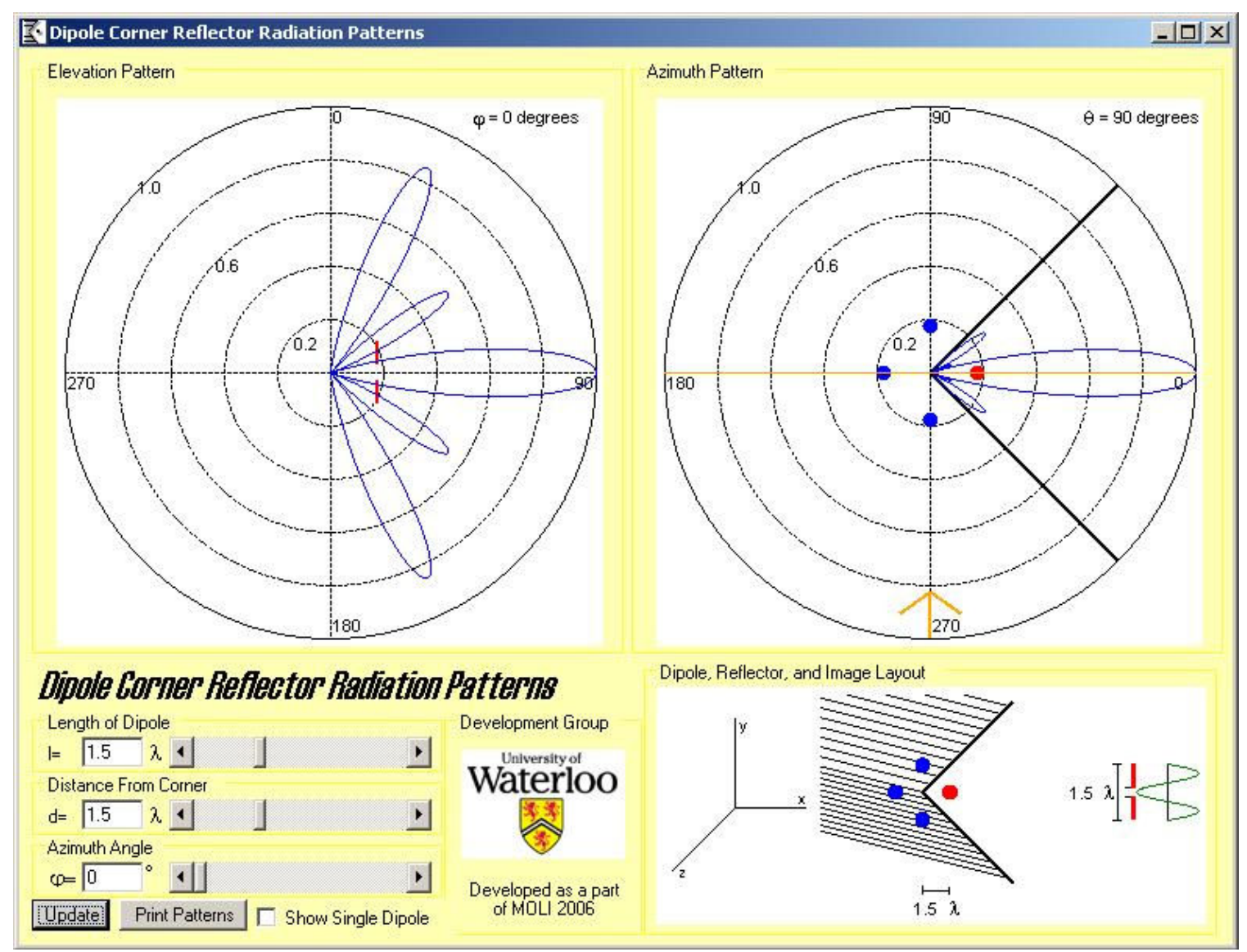

Figure 4: Single Dipole Inside an Infinite $90^{\circ}$ Corner Reflector Simulation Tool

An infinite $90^{\circ}$ corner reflector is a set of two ground planes positioned perpendicular to one another and extending out infinitely in length. The antenna is then placed with each side of the corner reflector forming a $45^{\circ}$ angle above and below the $x-z$ plane. By having the configuration as such, 3 dipole images are formed: one in the $x-z$ plane intersecting the negative $x$-axis, one in the $y-z$ plane intersecting the positive $y$-axis, and the third in the $y$ - $z$ plane intersecting the negative $y$-axis. The user has the ability to alter the length of the dipole, and the azimuth angle, and show the radiation pattern relative to the single dipole. In addition, the simulator is also capable of altering the dipole's distance from the corner of the corner reflector. As this parameter is altered, the distance of the real dipole and its three images are altered. All four of these distances are equidistant from the center of the corner reflector. The length of the dipole, its distance from the corner, and the current distribution along the dipole are all updated in the physical configuration window as changes are made to the parameters.

\section{Interface}

The success of any software tool is greatly affected not only by its functionality but also by its ease of use. By taking this into consideration when designing the user interface, the tool will hopefully get more use and be of greater value to the students using it. A key component to overcoming the learning curve to any tool is to have a similar look and feel across all tools of a particular product suite. In doing so, once the user is familiar with the first simulator, he or she has an understanding of the controls for all of the simulation tools. 
The icons and background colors for each module were also considered with great care. By using distinct icons and background colors for each simulator tool, students, teaching assistants, and lab instructors can easily differentiate between simulators at a single glance, allowing for less confusion when explaining information regarding each tool.

\section{Theory}

The azimuth and elevation patterns show plots of the antenna configuration's system factor. The system factor is calculated as shown in Equation 3.

$$
\text { SystemFactor }=\text { ArrayFactor } * \text { ElementFactor }
$$

The system factor is the product of the antenna configuration's array factor and the individual dipole element factor ${ }^{6}$. The array factor is dependent on parameters related to how the array of dipole elements or dipole images interact with each other. These parameters include the number of dipole elements, the element's weighting, the distance between dipoles or images, and the azimuth and elevation angles. The element factor on the other hand, is dependent on parameters that change the properties of the dipole. These parameters include the dipole length, and the azimuth and elevation angles. For the azimuth pattern, the elevation angle has been fixed at $90^{\circ}$ for all calculations and the azimuth angle is varied a full $360^{\circ}$ to calculate the pattern. For the elevation pattern, a fixed azimuth angle is selected by the student and the elevation angle is varied a full $360^{\circ}$ to calculate the pattern. Each pattern displayed in the viewing field thereby represents a 2-dimensional "slice" along the set azimuth and elevation angles to represent a full 3-dimensional image of the resulting radiation pattern. By plotting the system factor for each angle around the antenna configuration's origin, the relative antenna radiation pattern can be shown.

\section{Element Factor}

The element factor calculation is common among all four simulation tools. It has been normalized to the antenna's maximum range as shown in Equation $4^{7}$. The formula for the wave number $k$, used in the element factor calculation is shown in Equation 5.

$$
\begin{gathered}
E F=\left(\frac{\cos \left(\frac{k l}{2}\right) * \cos (\theta)-\cos \left(\frac{k l}{2}\right)}{\sin (\theta)}\right)^{2} \\
k=\frac{2 \pi}{\lambda}
\end{gathered}
$$

In Equation 4, $k$ represents the wave number, shown in Equation 5, where $k$ is inversely proportional to the wavelength, $l$ is the total length of the dipole antenna (in terms of wavelength), and $\theta$ is the elevation angle. Note that the element factor equation is independent 
of both the azimuth angle, $\phi$, and the wavelength, $\lambda$. The wavelength independence is a result of the cancellation from the dipole length component and the wave number. This indicates that the element factor radiation pattern is independent of the wavelength of the signal being propagated, and that the element factor's contribution to the azimuth pattern will always be uniform for every angle of $\phi$ since the elevation angle is fixed when calculating the azimuth pattern.

\section{Array Factors}

The array factor calculation is different for each antenna configuration. The array factor for the single dipole antenna is simply unity since it has no interaction with other dipole elements. For the other configurations, the array factor calculation is more involved. The array factor calculation for the dipole array is shown in Equation $6^{8}$.

$$
A F=I_{o}+\sum_{m=1}^{N / 2} 2 * \text { weight }(m) * I_{o} * \cos (2 \pi * d * m * \sin (\theta) * \sin (\phi))
$$

In this calculation, it is assumed that all elements are of equal length, equidistant along the $y$-axis and the dipoles oriented in the $z$-direction. The center dipole is situated at the origin and is used as the reference point. $I_{o}$ represents the normalized current, for all calculations it can be assumed to have a value of unity. $N$ is the total number of dipoles minus the center dipole. This value is divided by two in the summation because each pair of elements equidistant from the origin contributes equally to the array factor so each element in the summation is multiplied by two to take this into account. The weighting of each element is decided by the parameter selected by the user and is governed by Equation 1 and Equation 2. The parameter $d$ represents the distance between the dipoles. Finally, $\theta$ represents the elevation angle and $\phi$ the azimuth angle.

For the array factor calculation for the single dipole horizontally positioned above an infinitely flat ground plane it is assumed that the ground plane is situated in the $y$ - $z$ plane and the dipole is parallel to the ground plane with its center on the $x$-axis, and the height of the dipole from the ground plane is measured along the $x$-axis. The array factor calculation for the flat ground plane configuration is shown in Equation $7^{9}$.

$$
A F=2 * \sin (2 \pi * h * \sin (\theta) * \cos (\phi))
$$

The calculation is multiplied by two because there is both the real dipole and the dipole image created by the ground plane contributing equally to the array factor. The parameter $h$ is the height of the dipole (and implicitly the dipole image) from the ground plane, and $\theta$ and $\phi$ are the elevation and azimuth angles, respectively. Since the radiation pattern only exists on the side of the ground plane with the real dipole, points calculated on the image side of the ground plane are reflected in the ground plane to appear on the real side of the ground plane.

The array factor calculation for the single dipole inside of the $90^{\circ}$ infinite corner reflector is similar to the flat ground plane in that it can be calculated using image theory. By situating the corner reflector so that it creates a $45^{\circ}$ angle above and below the $x-z$ plane, and placing the dipole inside the corner reflector in the $x-z$ plane with its center intersecting the $x$-axis, three dipole images are created. These can be seen in Figure 4. Two of the images are in anti-phase, 
while the third is in phase with the original dipole. By solving for the two elements intersecting the $x$-axis, a resultant equivalent image appears at the origin. This element can then be used with the images intersecting the $y$-axis as a three element linear array to solve for the resulting array factor. This is performed in Equation $8^{10}$.

$$
A F=2 * I_{o} *[\cos (2 \pi * h * \sin (\theta) * \cos (\phi))-\cos (2 \pi * h * \sin (\theta) * \sin (\phi))]
$$

The parameters in the calculation include the normalized current $I_{o}$ which is set to unity, $h$ which represents the distance of the dipole (and implicitly the images) from the origin, and $\theta$ and $\phi$ which represent the elevation and azimuth angles respectively.

\section{Current Distribution}

To calculate the current distribution along the dipole, the dipole was assumed to be an ideal center-fed dipole. The total length of the dipole was assumed to be much greater than the diameter of the dipole, and the dipole is perfectly straight, meaning that there is no flaring. It was further assumed that the current distribution was time invariant. Based on these assumptions and simplifications, Equation 9 can be used to calculate the current distribution ${ }^{10}$.

$$
I(x)=I_{o} * \sin [k(l-|x|)]
$$

For the current distribution calculation, $I_{o}$ is maximum amplitude of the current, $k$ is the wave number as calculated in Equation 5, $l$ is half the length of the total dipole, and $x$ is position along the dipole ranging from $-l$ to $l$.

\section{Verification and Testing}

To verify that the simulation tools are accurate, in-laboratory testing and comparisons to other sources were performed. The main form of testing consisted of verifying the simulation tool's radiation patterns against those of radiation patterns in textbooks for the same configuration parameters ${ }^{12-14}$. In-laboratory testing for the single dipole configuration was performed by comparing experimental data collected by students in the ECE 471 lab course against the simulated data. The patterns produced by the simulation tools were identical to the ideal plots given in the reference texts, and provided a very good approximation of the experimental data collected.

\section{Simulator Availability}

The complete online dipole antenna simulation package was first released for use during the spring 2006 school term. Access to the simulators was first restricted only to those students taking the ECE 471 course during the term. The simulation tools were demonstrated in tutorial sessions and students were required to use the tools to complete a pre-lab study prior to attempting the antenna laboratory. A total of 96 students utilized the simulation tools. Although no formal statistical data was collected, the simulators were received well by students based on informal feedback. The next course offering for ECE 476 is during the winter 2007 term, and 
the next course offering for ECE 471 will be during the spring 2007 term. These terms will provide an opportunity to formally study the usage of the tools.

The simulators are now available to the members of the Microsoft Developer Network Academic Alliance (MSDN AA) for download and critiquing at www.moli.uwaterloo.ca/antennasim. The download is available as a single compressed file. Once extracted the folder content appears as shown in Figure 5.

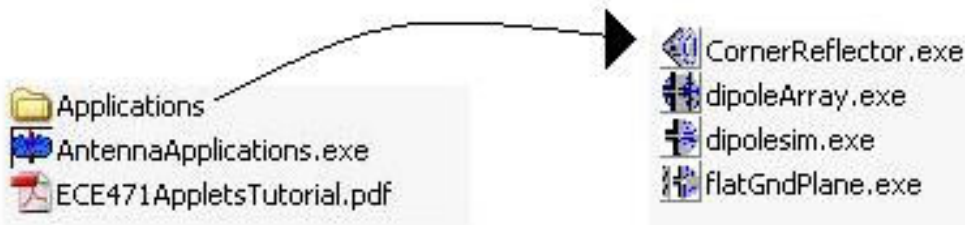

Figure 5: Simulation Package Directory Structure

AntennaApplications.exe is the main executable file. It launches an opening splash screen followed by the main menu. ECE471AppletsTutorial.pdf is the accompanying documentation for the simulation tools; it outlines all of the features for each of the simulators and describes how to use the controls. Finally, the applications folder contains the four simulator executable files. These files may be accessed via the main menu screen. To use the main menu, the user must maintain the same relative directory structure between the main menu and the Applications folder.

\section{Conclusions and Future Work}

This paper has shown that it is feasible to develop inexpensive, feature-rich antenna simulator tools tailored towards specific course material using existing tools and technologies. The simulators described are capable of providing students with virtual hands-on-learning prior to entering a laboratory for "real" hands-on-learning. This allows students to overcome the initial learning curve on new material and prepares them to ask more focused questions during lab sessions.

Now that the simulators have been used by students in class during the initial phase of usage, the simulators are available for use and critique within the academic community to members of the MSDN AA. Feedback from other established institutes is welcome as it can lead to further improvements and modifications of the tools for more effective use within the classroom and in classrooms across the world.

\section{Acknowledgements}

The authors of this paper would like to thank Microsoft Canada and the Microsoft Online Learning Initiative (MOLI) for funding this project. 


\section{Bibliography}

1. S. M. Wentworth, Fundamentals of Electromagnetics with Engineering Applications, Wiley, 2004.

2. Linear Antenna Interactive Java Applets, http://www.amanogawa.com/antenna.html, 07 Dec 2006.

3. SuperNEC - EM Antenna Simulation Software \& Design, http://www.supernec.com/, 07 Dec 2006.

4. ComApps.com, http://www.comapps.com/tonyt/Applets/Antennas/Antenna.html, 07 Dec 2006.

5. Analyze Math - Antennas, http://www.analyzemath.com/antennas.html, 07 Dec 2006.

6. F. T. Ulaby, Fundamentals of Applied Electromagnetics, Prentice-Hall, Inc., 2001, p. 375.

7. F. T. Ulaby, Fundamentals of Applied Electromagnetics, Prentice-Hall, Inc., 2001, p. 360.

8. "Antennas and Wireless Systems," class notes for ECE 476, Electrical and Computer Engineering Department, University of Waterloo, Waterloo, ON, Canada, Winter 2006, p. 72.

9. R. S. Elliott, Antenna Theory and Design, Prentice-Hall, Inc., 1981, p. 68.

10. E. Spike, C. Santillan Rios, ECE 471 Corner Reflector Tutorial Notes, tutorial notes for ECE 471, Electrical and Computer Engineering Department, University of Waterloo, Waterloo, ON, Canada, Winter 2006.

11. L. C. Shen, J. A. Kong, Applied Electromagnetism, PWS Publishing Company, 1995, p. 227.

12. F. T. Ulaby, Fundamentals of Applied Electromagnetics, Prentice-Hall, Inc., 2001, p. 347.

13. H. P. Williams, Antenna Theory and Design, Sir Isaac Pitman \& Sons Ltd, 1966, p. 128.

14. "Antennas and Wireless Systems," class notes for ECE 476, Electrical and Computer Engineering Department, University of Waterloo, Waterloo, ON, Canada, Winter 2006, p. 78-79. 